\title{
¿Se trabajan de forma interdisciplinar música y matemáticas en educación infantil?
}

Rocío Chao Fernández

María Dorinda Mato Vázquez'

Andrea Mª́pez Chao'

\section{Resumen}

El propósito de esta investigación es analizar si se trabajan de manera interdisciplinar las materias de música y matemáticas en los primeros años de escolaridad. Opción metodológica que en los últimos años está recibiendo un gran empuje en esta etapa educativa, en contraposición a los modelos tradicionales. La muestra está formada por 360 profesores de educación infantil y educación musical de colegios públicos y concertados de la provincia de $\mathrm{A}$ Coruña (Galicia, España). Para llevar a cabo el análisis se diseñó un cuestionario que se aplicó en noviembre de 2013. Los resultados reflejan que la mayoría de profesores pertenece a centros públicos y le otorgan la misma importancia a las asignaturas de música y matemáticas. Consideran que es importante trabajarlas de forma interdisciplinar, pues de este modo mejora la formación integral del alumnado. Sin embargo, no se sienten capacitados para abordar el proceso de enseñanza-aprendizaje de este modo. En la mayoría de los casos son distintos profesores quienes imparten ambas materias y no programan ni utilizan materiales y actividades de forma conjunta, aunque evalúan de forma interdisciplinar. Por último, en la discusión, se realizan ciertas consideraciones finales que ponen de manifiesto el porqué es importante la investigación, pues los resultados obtenidos nos permiten conocer la realidad de nuestras aulas, y nos inducen a afirmar la necesidad de la formación del profesorado para dar impulso a la enseñanza-aprendizaje de música y matemáticas de educación infantil a través de la interdisciplinariedad.

\section{Palabras clave}

Música - Matemáticas - Interdisciplinaridad - Educación infantil - Enseñanza-aprendizaje. 


\section{Are music and mathematics worked on in an interdisciplinary way during early childhood education?}

Rocío Chao Fernández

María Dorinda Mato Vázquez

Andrea Mán López Chao'

\begin{abstract}
The purpose of this investigation is to analyse whether music and mathematics are subjects taught in an interdisciplinary way in the earliest school years, a methodological option that had a great impact in the past few years on this educational stage, opposed to the traditional methods. The sample is made up by 360 teachers of early childhood education and musical education from both public and private schools in the province of a Coruna (Galicia, Spain). To carry through the analysis, we designed a questionnaire that was applied in November 2013. The results show that most of the teachers belong to public schools and give the same importance to both subjects, music and mathematics. They consider that it is important to work on them in an interdisciplinary way, as that improves the integral academic training of students. In the majority of the cases, there exists a different teacher for each subject, and they do not program or use materials and activities jointly, although they do evaluate in a interdisciplinary manner. Lastly, in the discussion we make certain final considerations that evidence what makes investigation important, as our findings allow us to know what is happening in the classrooms and lead us to confirm the need for teacher education to give an impulse to the teaching-learning process of music and mathematics through interdisciplinarity.
\end{abstract}

\section{Keywords}

Music - Mathematics - Interdisciplinarity - Early childhood education - Teaching-learning. 


\section{Introducción}

Coincidimos con Castro (2008) cuando asevera que la educación infantil es una etapa fundamental en el proceso de formación y desarrollo integral de la persona, que debería atender a condiciones materiales, atenciones físicas, relaciones humanas afectivas y estímulos que activen su desarrollo físico y mental. Además, numerosos estudios, entre los que se encuentra el realizado por Gómez Martín (2012), aseguran que no se debe ignorar una etapa tan fundamental en la formación de una persona y que tanto la condicionará en el futuro. Es por eso que la escuela es un contexto enriquecedor en el desarrollo de los niños y niñas al permitirles construir su personalidad, ampliar sus experiencias y favorecer su socialización.

Por tanto, basándonos en la corriente constructivista, para llegar a una educación de calidad, el proceso de enseñanza debe ser percibido y llevado a cabo como un procedimiento dinámico, participativo e interactivo. Estamos de acuerdo con Alsina y Escalada (2008) cuando afirman que con el fin de construir conocimiento deben buscarse métodos para que los/as niños/as se involucren en la resolución de problemas, llevándose a cabo un aprendizaje autónomo y auto dirigido. Por tanto es importante crear grupos de aprendizaje en los que las personas trabajen de manera conjunta e interdisciplinar. Para ello será necesario plantear actividades que logren despertar el interés del alumnado y que permitan la observación y la experimentación.

En paralelo consideramos que la formación permanente del profesorado es un requisito indispensable para el desarrollo profesional de todo docente, y la preocupación por saber cómo, de qué modo, con qué modelos y si el conocimiento se actualiza, es cada vez más común en los ámbitos de investigación y evaluación educativa, pues la calidad de los procesos de enseñanza y aprendizaje y la formación del profesorado se materializan en el aula.
En la formación del profesorado de educación infantil, en los últimos años, irrumpen con fuerza las didácticas específicas, que conjugan el conocimiento didáctico con el contenido científico específico. Estos conocimientos aportan un marco teórico capaz de dirigir la formación del profesorado hacia un planteamiento analítico hiperespecializado, en el que cada disciplina contribuye al conocimiento. Esta opción puede ser criticada por algunos profesionales que alegan conducir a la desconexión de saberes, sin aportar las necesarias pautas de integración. Pero, también se plantea la necesidad de utilizar el diálogo disciplinar, entendido como un espacio que permita articular el conocimiento de las diferentes materias en el estudio de los hechos del mundo. Es decir, se produce un constante ir y venir entre las diferentes ópticas (BONIL et al., 2004). Por tanto este fenómeno no puede estudiarse desde una, sola perspectiva pues la conjunción de varias posee un valor añadido que supera la suma de las partes. Desde esta perspectiva hay que superar dificultades estructurales y de índole personal y buscar planteamientos formativos que pongan en juego el diálogo disciplinar en el aula de educación infantil. Es decir, que la formación del profesorado de los primeros cursos tome medidas hacia la interrelación entre disciplinas.

Para dar respuesta a esta inquietud seleccionamos como objeto de nuestra investigación dos disciplinas presentes en la vida de los niños y niñas muy importantes para su desarrollo: la música y las matemáticas; ambas materias tienen un papel relevante ya desde la educación infantil. La razón de nuestro análisis tiene que ver con el hecho de que la matemática es considerada una de las asignaturas con peor rendimiento en la escuela. Sin embargo, su estudio es fundamental; no en vano contribuye al desarrollo del pensamiento lógico, a la exactitud y al desarrollo de la percepción espacial (ILARI, 2002; THIESSEN, 2004). Por otro lado, la música tiene muchos 
beneficios para el desarrollo cognitivo de los niños/as (FERNÁNDEZ BRAVO, 2006) y consideramos que ambas disciplinas guardan una relación mucho más estrecha de lo que podría pensarse a primera vista (FAUVEL; FLOOD, 2003; LIERN; QUERALT, 2008) además del beneficio que se obtiene en la formación integral del individuo si se trabajan de manera conjunta. Partiendo de esta premisa, creemos que se debe prestar atención a la enseñanzaaprendizaje vinculando ambas materias. Para realizar esta investigación se ha diseñado un cuestionario para conocer si en las aulas de educación infantil se trabajan conjuntamente música y matemáticas y la metodología empleada por el profesorado para hacerlo.

\section{Música y matemáticas}

La bibliografía revisada nos ha permitido comprobar que la relación entre música $\mathrm{y}$ matemáticas ha sido muy estrecha a lo largo los tiempos; de hecho diferentes autores han realizado las aportaciones al campo de estas dos disciplinas (ARBONÉS; MILRUD, 2011; PERALTA, 1998; IBARRIAGA, 2010).

La música es un elemento fundamental, de manera especial en la primera etapa del sistema educativo: les gusta mucho a los alumnos, les proporciona seguridad emocional y confianza, comparten canciones con los compañeros y compañeras, lo que les proporciona un clima de ayuda, de colaboración y de respeto. Estamos de acuerdo con Álvarez, Mollá y Estébanez (2009) cuando aseguran que la educación musical fomenta el desarrollo de la vida emocional, de las habilidades creativas y expresivas, de la estética y la sensibilidad en general, de la flexibilidad de pensamiento y de la organización temporal. Además creemos firmemente que la música es una estrategia estimuladora del proceso de enseñanzaaprendizaje muy eficaz, coincidiendo con investigaciones, como las realizadas por Chao, Mato y Ferreiro (2014), Levitin y Álvarez (2008), Pérez Adalguer y Leganés (2012), etc. que demuestran que la educación a través de la música enriquece la experiencia del aprendizaje en general. Un ejemplo de ello son los estudios llevados a cabo por el profesor Levitin (2011), quien afirma que mediante el uso de la música nuestro cerebro produce un aprendizaje más acelerado y significativo.

Es una realidad que la etapa de alfabetización del niño y niña se ve más estimulada a través de la música (WINKLER et al. 2009); sin embargo, en muchas ocasiones, la educación musical tiende a carecer de relevancia bajo los ojos del profesorado, sobre todo si se compara con otras materias, a las que consideran fundamentales, como es el caso de las matemáticas. Valoramos su importancia, entre otras cosas, porque son imprescindibles en este mundo tecnológico en el que vivimos, y además, lo son en todos los niveles educativos y en el día a día de cada quien. No obstante suelen ser de las materias que menos gustan a los escolares, las que más suspenden y las que evitan, si pueden, al llegar a cursos superiores. El conocimiento matemático les sirve a los más pequeños y pequeñas para comprender y manejar la realidad social en la que van a insertarse. Además, contribuye a desarrollar la comunicación, interpretar el mundo en el que viven y favorecer el desarrollo de conocimientos para poder razonar, aplicar estrategias de análisis y resolución de problemas. En defınitiva, en la etapa de educación infantil, aprender a resolver problemas (entendidos como situaciones que no podemos resolver algorítmicamente o automáticamente y que precisan de una investigación y un pensar las cosas) es la finalidad básica que se debe perseguir (GREGORIO GIRLES, 2002). Pero como indica Canals i Tolosa (2001), en esta etapa tan particular es imposible trabajar los contenidos matemáticos aislados de otras actividades, ya que las matemáticas que se deben presentar en el aula deben estar relacionadas también con la educación sensorial y del lenguaje, con la psicomotricidad, la plástica, los cuentos, la música, y el conocimiento del medio. 
Investigaciones recientes afirman que el interés por las matemáticas, disminuye conforme los alumnos avanzan de curso (MAT0; MUÑOZ, 2010) y que los primeros años son fundamentales para la adquisición del desarrollo del pensamiento matemático, y principalmente la prevención de actitudes negativas (MATO, 2010). Los estudios anteriores también demuestran que una de las causas por las que los alumnos no se interesan por las matemáticas es la metodología empleada. Nuestra investigación puede ser de gran ayuda para los profesionales de la educación al pretender trabajar ambas materias de manera relacionada, divertida y dinámica. De hecho, Gómez Martín (2012) reconoce que las investigaciones llevadas a cabo en las dos últimas décadas del siglo XX sobre las matemáticas y su aprendizaje a edades tempranas llevó a muchos países a replantearse los contenidos. En particular, la prestigiosa National Council of Teachers of Matematics (NCTM) americana decidió en 2000 reformar la programación de la etapa de infantil.

Al respecto, Fernández-Carrión (2011) corrobora que al apoyarse de manera interdisciplinar las matemáticas y la música se establecen similitudes con la realidad que se vive, se acerca a la cotidianidad de la vida, se muestra más útil, práctica, dinámica y por encima de todo, se presenta motivadora. Por razones como estas, a lo largo del trabajo, se abordará la relación entre las dos disciplinas, profundizando en los conceptos comunes que estas presentan y haciendo un especial hincapié en la influencia existente entre ambas.

El vínculo entre estas disciplinas tiene sus inicios en la antigua Grecia. Los pitagóricos (siglo VI a.C.) fueron los primeros en dotarnos de una teoría filosófica sobre la música, la conocida armonía de las esferas, crearon la escala diatónica y consideraron que la esencia del conocimiento estaba compuesta por siete artes liberales: Geometría, Aritmética, música, Astronomía, Gramática, Dialéctica y Retórica. La posición de la música como un subconjunto de las matemáticas (Quatrivium) permaneció durante la Edad Media.
Años más tarde, Leonardo Fibonacci (1170-1250) inventó la Sucesión de Fibonacci, que habla del número áureo o número de oro. Dicho número juega un papel muy importante en la Geometría y en la Estética. Otros compositores como Bártok, Messiaen y Stockhausen, escribieron obras cuyas unidades formales se relacionaban (a propósito) con la sección áurea. También aparece en las estructuras formales de las sonatas de Mozart, en la Quinta Sinfonía de Beethoven, en obras de Schubert y Debussy o en la coral situada al final de Kunst der Fuge de Johann Sebastian Bach.

En esta línea, un reconocido filósofo dedicado, principalmente, al campo de la teología, las matemáticas y la música fue Marin Mersenne (Francia,1588-1648). Su mayor contribución al campo de la teoría musical y los instrumentos musicales fue la sugerencia de que $\sqrt{\sqrt{\frac{2}{3-\sqrt{2}}}}$ era la razón principal de un semitono.

Otro autor, no menos conocido es Gottfried Leibniz (1646-1716), pionero en el desarrollo de la lógica matemática, y uno de los precursores de los ordenadores. Se le atribuye, así mismo, el descubrimiento del cálculo infinitesimal, independientemente de Newton. Su notación se halla desde entonces en uso general. Considera la música como "un ejercicio aritmético ocultado del alma, que no sabe que está contando"(CHA0, 2005, p.34).

Por su parte, Leonhard Euler (1707-1783) estableció un criterio de armonicidad de cualquier intervalo o acorde: cuánto más pequeños sean los números que expresan la relación de vibración de dos notas, éstas serán más consonantes. En relación con este matemático, Rameau (16831764) y D'Alembert (1717 - 1783) desarrollaron que el grado de armonicidad era distinto del agrado o medida estética.

Nos parece importante mencionar que el gran músico Wolfgang Amadeus Mozart (17561791) era un gran aficionado a las matemáticas, y para escribir valses cifró el famoso juego de dados musical. Se trata de 176 compases colocados en dos tablas de 88 elementos cada 
una. Otro autor de renombre es el matemático George Birkhoff (1884-1944) quién propuso la fórmula $\mathrm{M}=0 / \mathrm{C}$ para expresar la medida estética como el efecto de la densidad de las relaciones de orden comparadas con la complejidad. En lo que a música se refiere, aplica esta fórmula a los acordes diatónicos, armonía y melodía así como a la calidad musical en la poesía.

De forma más reciente, el compositor y arquitecto Iannis Xenaquis (1922-2001) concibió y desarrolló el sistema UPIC (Unidad Polyagógica Informática del centro de estudios en matemáticas y música automatizada)... Propuso la utilización de modelos matemáticos en la composición musical y algunos de los procedimientos utilizados en sus composiciones incluyen la teoría de probabilidades, la teoría de juegos, la teoría de grupos y el álgebra booleana.

Los ejemplos ilustrados nos permiten deducir que la estrecha relación entre ambas disciplinas ha proporcionado herramientas muy útiles a lo largo de la historia. Muchas de ellas pueden resultar de gran ayuda para dar contenidos matemáticos $\mathrm{y}$, especialmente musicales, teniendo en cuenta el interés que despierta esta última entre los niños y niñas de educación infantil. Sin ir más lejos, la relación existente entre ambas se manifiesta en la lectura de una partitura. En ella observamos numerosos conceptos matemáticos como la representación del pentagrama, el formato por cinco líneas y cuatro espacios y las notas musicales, y su colocación en el mismo.

Si nos adentramos en el campo de la educación, hay numerosos pedagogos, que tratando de desarrollar su método musical, trabajaron transversalmente diversos conocimientos del área de las matemáticas (CHA0, 2005). Por ejemplo, Jaques Dalcroze (1865-1950) emplea cartones para la enseñanza de las notas musicales que se caracterizan por ser de la misma forma, tamaño y color.

En relación con lo mencionado anteriormente, Carl Orff (1895-1982) creó una serie de instrumentos para desarrollar su metodología entre los que destacan los xilófonos y carillones, compuestos por un número determinado de piezas rectangulares que representan los diferentes sonidos de la escala musical. Se trata de instrumentos de pequeña percusión a través de los cuales se pueden trabajar las formas y figuras geométricas (el círculo, el triángulo, la forma ovalada), así como su tamaño y cantidad (el número de piezas que compone un instrumento).

Por su parte, Martenot (1898-1980) es conocido por los juegos que ideó para trabajar las distintas capacidades musicales. Son ejercicios en los que se aborda el tema de la velocidad (rápido-lento), la altura de las notas (alto-bajo) y la duración de los timbres y sonidos mediante el tiempo y la memoria.

En esta misma línea, Justine Ward (1880-1975) desarrolló la notación cifrada la cual sustituye las notas musicales por números del uno al siete. A Kodály (1882- 1967) se le atribuye la representación de las notas musicales mediante determinados gestos manuales, en los cuales se trabaja la altura, ya que dependiendo de ella los sonidos serán más graves (bajos) o más agudos (altos).

Edgar Willems propone la realización de ejercicios basados en reconocer y emparejar sonidos fuertes y débiles, graves y agudos, movimientos rápidos y lentos, etc. $\mathrm{Al}$ igual que en muchos de los métodos descritos anteriormente, se le da al ritmo un papel protagonista, así como a los ejercicios de audición interior, en los que los niños y niñas deben ser capaces de contar en voz baja respetando el tiempo. Resalta el papel de instrumentos de pequeña percusión a través de los cuales trabaja el tamaño (grande-pequeño) y las formas de los mismos; el carillón intratonal, el cual los niños y niñas deben clasificar y ordenar correctamente; y los tubos sonoros, los cuales se utilizan mediante movimientos circulares de mayor y menor velocidad, lo que permite conseguir la serie armónica.

Otro de los autores a mencionar es Jos Wuytack (1935) que se caracteriza por ser el creador del musicograma. Se trata de un procedimiento en el que podemos visualizar 
lo que oímos. Está formado, principalmente, por dibujos de colores, con distintas formas y figuras geométricas, símbolos, etc.

Tras observar la gran cantidad de estudiosos de la música y las matemáticas que han trabajado conjuntamente estos dos ámbitos, podemos observar los grandes beneficios de esta simbiosis. En definitiva, queda patente la gran utilidad de trabajar estas materias de forma interdisciplinar en el aula.

\section{Objetivo}

El objetivo que nos proponemos con esta investigación es averiguar si en las aulas de educación infantil se trabajan matemáticas y música interdisciplinariamente y cómo se hace.

\section{Participantes en el estudio}

La muestra invitada está formada por setecientos profesores seleccionados de forma aleatoria, siendo la muestra aceptante de 360 profesores pertenecientes a colegios públicos y concertados de la provincia de A Coruña de educación infantil y educación musical.

\section{Instrumento}

Para realizar la investigación se diseñó un cuestionario mixto compuesto por respuestas abiertas y cerradas. Éste fue cubierto por el profesorado de educación infantil y educación musical.

El cuestionario se compone de diversos ítems, agrupados en cuatro grandes bloques:

$1^{\circ}$ : Información sobre el profesorado: tipo de centro en el que imparten docencia, sexo, edad, estudios que poseen y especialidad, años de experiencia docente.

\section{$2^{\circ}$ : Carácter interdisciplinar de las materias}

- ¿Es el mismo profesor el de música y el de matemáticas?

- $\mathrm{Si}$ es el mismo profesor, realiza conjuntamente o de forma separada para ambas asignaturas las programaciones, actividades, materiales, recursos, evaluación.

-Si son distintos profesores, realizan conjuntamente o de forma separada programaciones, actividades, materiales, recursos y evaluación.

3०: Metodología utilizada en el aula

- ¿Utiliza las matemáticas y la música de forma interdisciplinar?

- ¿Cómo lo hace?

-Para el desarrollo de sus clases, ¿qué metodología emplea?

-¿Qué recursos emplea?

$4^{0}$ : Valoración

- ¿Se siente preparado para trabajar la música y las matemáticas interdisciplinariamente?

- ¿Qué valora más la música o las matemáticas?

- ¿Se siente satisfecho con su trabajo?

- ¿Considera que la enseñanzaaprendizaje mejora trabajando las dos áreas conjuntamente?

Para contestar las preguntas cerradas se debía marcar con una cruz o rodear con un círculo el número de la respuesta o respuestas elegidas. En las preguntas abiertas se requería un mayor esfuerzo, puesto que era necesaria la redacción por parte de las personas encuestadas.

\section{Procedimiento}

Los cuestionarios fueron repartidos entre el profesorado de educación infantil y de música en diferentes escuelas públicas y concertadas $^{1}$ de la provincia de A Coruña, en el mes de noviembre del año 2013. Los profesores que participaron en el estudio respondieron

1- Las escuelas públicas son aquellas que están sostenidas con fondos públicos e imparte los ciclos educativos obligatorios (desde los 6 hasta los 16 años). La enseñanza es laica y gratuita.Las escuelas concertadas son centros privados que establecen un acuerdo con la Administración Pública para ser subvencionados con fondos públicos. Esto les obliga a adaptarse a ciertos condicionantes establecidos por el gobierno, como límite de alumnos por clase, fechas, admisiones etc. Suelen estar regidos por religiosos con ideario propio y, aunque la enseñanza está subvencionada por el Estado, el resto de servicios del colegio deben pagarlos los padres (comedor, transporte, actividades extraescolares...) 
a los cuestionarios de forma individual, dejando un margen de tiempo de una semana. Posteriormente se procedió al análisis del instrumento de evaluación atendiendo a los diferentes ítems descritos anteriormente. Se realizó un análisis cualitativo de los bloques de metodología y carácter interdisciplinar de las materias y un análisis cuantitativo de los apartados de información acerca de los sujetos encuestados y valoración. En el último caso los datos obtenidos se recogieron en gráficas.

Debemos aclarar que el bloque de información sobre el profesorado nos ha servido para conocer la muestra de sujetos que han cubierto el cuestionario, por lo que los datos correspondientes aparecen en el apartado de participantes de la investigación.

\section{Resultados}

A continuación, realizamos una exposición y breve descripción de los resultados obtenidos tras la aplicación del cuestionario.

\section{Información sobre el profesorado}

- Del total de la muestra aceptante 328 profesores pertenecen a centros públicos y 32 a concertados.

En lo referente a la edad, la mayor parte de la muestra está entre los 30 y 50 años. Hay que destacar que hubo 72 personas que no respondieron a este ítem (Gráfico 1).

En relación con los estudios, la gran mayoría son diplomados (210) y 104 licenciados.

Gráfico 1- Edad del profesorado

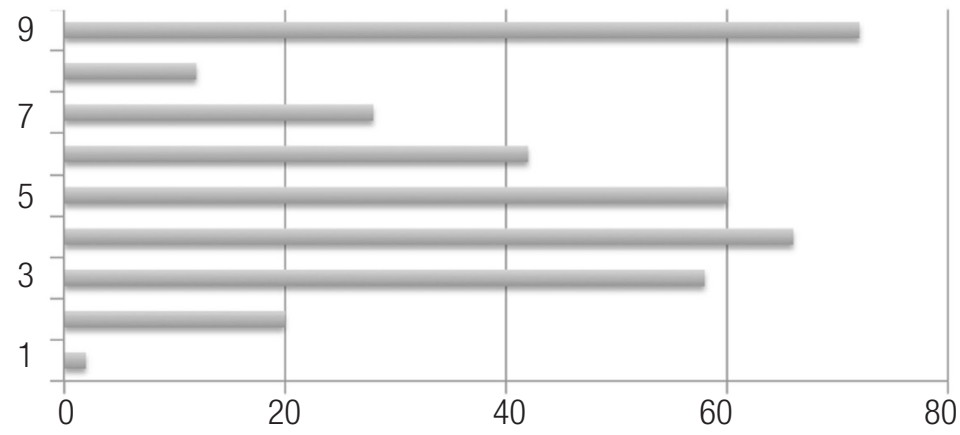

$$
\begin{aligned}
& \text { Edad } \\
& 20-25 \\
& 26-30 \\
& 31-35 \\
& 36-40 \\
& 41-55 \\
& 46-50 \\
& \text { 51-55 } \\
& \text { Más de } 56 \\
& \text { NC }
\end{aligned}
$$

Fuente: elaborado por los autores para este estudio

36 personas declaran poseer otros estudios, aunque no especifican cuáles y 30 no contestan. Curiosamente, del total de la muestra, tan solo 20 profesores estudiaron la especialidad de educación musical.

\section{Carácter interdisciplinar de las materias}

A la pregunta de si es el mismo profesor el de música y el de matemáticas, el 15\% de las respuestas de este ítem son afirmativas, frente a un 85\% que responde negativamente (Gráfico 2).
Al 15\% que declara ser el mismo profesor el que se encarga de impartir música y matemáticas, se le preguntó si realiza conjuntamente o de forma separada para ambas asignaturas las programaciones, actividades, materiales, recursos y evaluación. Como se puede observar en la tabla 1, el 15\% afirma realizar las programaciones y utilizar los mismos materiales y recursos para trabajar las dos asignaturas, frente a un 85\% que no lo hace. Con respeto a las actividades, sorprendentemente, el 90,9\% de los encuestados afirma utilizar las 
Gráfico 2- ¿Es el mismo profesor el de música y el de matemáticas?

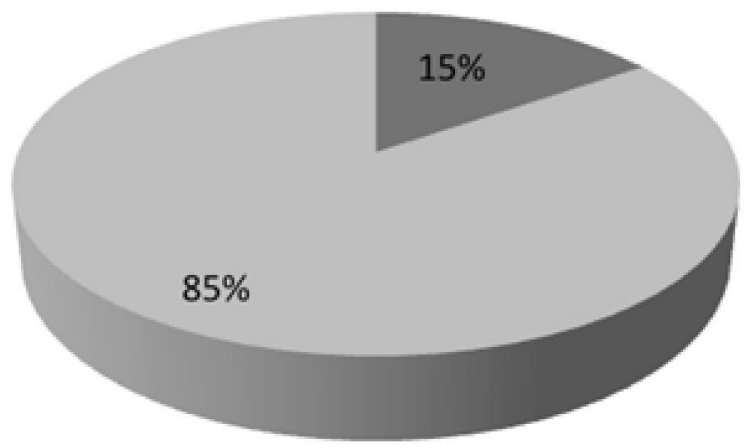

Fuente: elaborado por los autores para este estudio

mismas actividades para ambas materias, en contraposición de un 9,1\% que no lo hace. Por último, con respecto a la evaluación, el 81,8\% del total de la muestra valora conjuntamente dichas asignaturas, frente a un $18,2 \%$ que lo hace por separado.

Tabla 1- Si es el mismo profesor, ¿realiza las programaciones de manera interdisciplinar?

\begin{tabular}{c|c|cc}
\hline & & $\mathrm{Si}$ & No \\
\hline Programaciones & $15 \%$ & $85 \%$ \\
\hline Materiales & $15 \%$ & $85 \%$ \\
Recursos & $15 \%$ & $85 \%$ \\
Actividades & $90,9 \%$ & $9,1 \%$ \\
Evaluación & $81,8 \%$ & $18,2 \%$ \\
\hline
\end{tabular}

Fuente: elaborado por los autores para este estudio

Al 85\% del profesorado que declaró ser distinto profesor el que se encarga de impartir música y matemáticas también se le preguntó si realizan conjuntamente o de forma separada las programaciones, actividades, materiales, recursos y evaluación. En este caso, según se aprecia en la tabla 2, el 12,5\% afirma realizar las mismas programaciones y utilizar los mismos recursos en ambas asignaturas, frente a un 87,5\% que no lo hace. En relación tanto a las actividades como a los materiales, el $100 \%$ de la muestra responde que emplea materiales y actividades específicas para cada materia. Por último, haciendo referencia a la evaluación, un 25\% de las personas entrevistadas contesta que realiza la misma evaluación, mientras que un 75\% no lo hace.

Tabla 2- Si es distinto profesor, ¿realizan las programaciones de manera interdisciplinar?

\begin{tabular}{c|cc:c}
\hline & Si & No \\
\hline Programaciones & $12,5 \%$ & $87,5 \%$ \\
\hline Materiales & $12,5 \%$ & $87,5 \%$ \\
Recursos & $0 \%$ & $100 \%$ \\
Actividades & $0 \%$ & $100 \%$ \\
Evaluación & $25 \%$ & $75 \%$ \\
\hline
\end{tabular}

Fuente: elaborado por los autores para este estudio

\section{Metodología utilizada en el aula}

A la pregunta de si utiliza las matemáticas y la música de forma interdisciplinar, el $60 \%$ de los encuestados considera que sí 
utilizan las matemáticas y la música de modo interdisciplinar, en contraposición al 40\% que declara utilizarlas de forma independiente (Gráfico 3). Del porcentaje que asegura emplearlas de forma interdisciplinar, apenas un 20\% afırma trabajar estos ámbitos de forma combinada.

En relación a cómo la llevan a cabo el 20\% explica que utiliza diferentes canciones y rimas para escribir los números.

Gráfico 3- Utiliza la música y las matemáticas de forma interdisciplinar

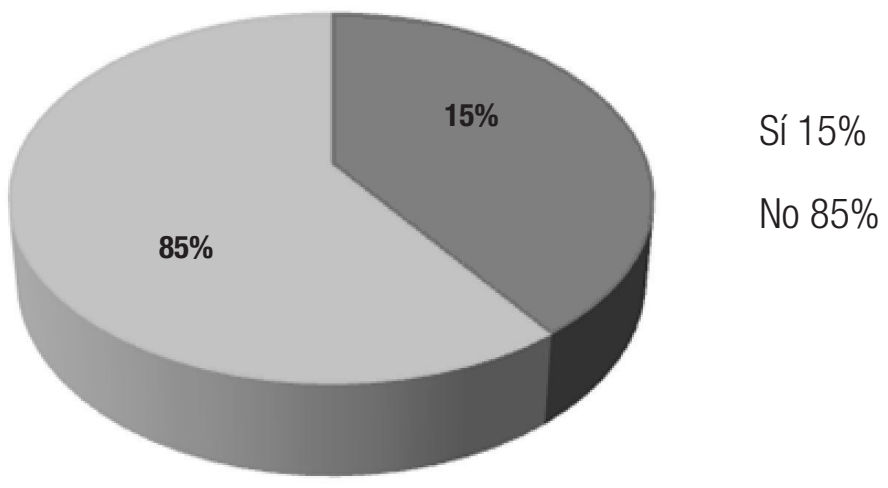

Fuente: elaborado por los autores para este estudio

En cuanto a la pregunta ¿qué metodología emplea para trabajar música y matemáticas en el desarrollo de sus clases? hay que destacar que al tratarse de una pregunta abierta varios profesores han expresado emplear varias metodologías combinadas. De este modo, según se aprecia en la tabla 3, el 50\% de los sujetos intenta llevar a cabo las actividades de forma lúdica para proporcionar un mejor aprendizaje al alumnado. Dentro de este porcentaje, el 35\% de las respuestas se postulan de cara hacia una enseñanza más participativa y a su vez, el 15\% especifica hacerlo de manera activa. El 25\% del total de la muestra afirma usar una metodología interdisciplinar, mismo porcentaje que aquellas que utilizan el aprendizaje significativo como base para el día a día en el aula. Por otro lado, el 15\% de las entrevistadas basan su trabajo en la teoría constructivista. Finalmente, se obtiene un porcentaje de apenas un 5\% en respuestas que hacen referencia al trabajo por proyectos, de forma individualizada, en equipo o partiendo de los intereses de los niños y niñas.

Tabla 3- ¿Qué metodología emplea para trabajar música y matemáticas?

\begin{tabular}{ccc}
\hline Metodología & $\vdots$ & $\%$ \\
\hline Forma lúdica & $\vdots 0 \%$ \\
\hline Enseñanza participativa & $\vdots$ & $30 \%$ \\
\hline Enseñanza activa & $\vdots$ & $15 \%$ \\
\hline Interdisciplinar & $25 \%$ \\
\hline Aprendizaje significativo & $\vdots$ & $25 \%$ \\
\hline Teoría constructivista & $15 \%$ \\
Trabajo por proyectos & $5 \%$ \\
\hline
\end{tabular}

Fuente: elaborado por los autores para este estudio

En referencia a la pregunta ¿Qué recursos emplea?, nuevamente se trata de una pregunta de respuesta abierta, por lo que en algunos casos han proporcionado diversos recursos. Así pues, como se percibe en la Tabla 4, el 75\% de la 
muestra afirma usar materiales proporcionados por el centro, alternándolos con otro tipo de recursos, en la mayor parte de los casos. Dentro de este porcentaje, un 20\% confirma recurrir específicamente a los materiales del aula. El 25\% restante de la muestra no especifica utilizar los materiales ni del centro ni del aula. Del total de la muestra, un 30\% de las docentes se apoya en las tecnologías de la comunicación (TIC), además de utilizar la pizarra digital interactiva (PDI) y otros recursos audiovisuales. Además, el 20\% de las entrevistadas afirma utilizar materiales editoriales tales como los libros del método además de recurrir diariamente al uso de fichas. Este porcentaje es superior al apenas 15\% de los encuestados que se encarga de realizar materiales didácticos de elaboración propia. Volviendo al total de la muestra, cabe resaltar que el 15\% recuerda los recursos que se pueden aprovechar del medio y de las salidas extraescolares y solo un 5\% destaca la participación de las familias y los especialistas. El 20\% hace referencia a los recursos necesarios para trabajar la música en el aula, basándose especialmente en instrumentos musicales. A su vez, otro 20\% trabajan el ámbito matemático centrándose en materiales y juegos de lógica-matemática.
Tabla 4- ¿Qué materiales emplea para trabajar música y matemáticas?

\begin{tabular}{c:c}
\hline Materiales proporcionados por el centro & $75 \%$ \\
\hline TICs & $30 \%$ \\
\hline Materiales editoriales & $20 \%$ \\
\hline Materiales elaboración propia & $15 \%$ \\
\hline Recursos que se pueden aprovechar del medio & $15 \%$ \\
\hline Salidas & $5 \%$ \\
\hline Recursos e instrumentos musicales & $20 \%$ \\
\hline Materiales y juegos de lógica-matemática. & $20 \%$ \\
\hline
\end{tabular}

\section{Valoración}

En relación a la pregunta de si se siente preparado para trabajar la música y las matemáticas interdisciplinariamente, sólo el 12\% afirma que su formación es suficiente, frente a un $88 \%$ que considera que no se sienten preparados (Gráfico 4). Cabe destacar la presencia de un 45\% de encuestados, dentro del grupo de personas que creen que su preparación es insuficiente, que añaden una observación sobre la inexistencia de experiencia en didáctica musical.

Gráfico 4- Se siente preparado para trabajar la música y las matemáticas de forma interdisciplinar

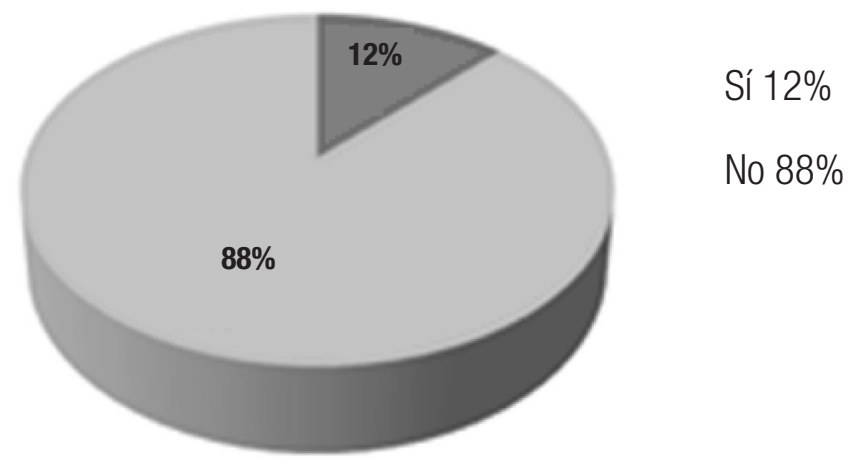

Fuente: elaborado por los autores para este estudio 
A la pregunta que hace referencia a si valora más la música o las matemáticas el $5,5 \%$ de la muestra considera que la música es más importante; el 16,5\% que son las matemáticas las más importantes, frente al $34,4 \%$ que considera que las dos asignaturas poseen la misma importancia, desarrollando así determinadas habilidades de los niños y niñas. Cabe destacar que un porcentaje muy elevado $(43,6)$ no contesta a esta pregunta (Tabla 5).

Tabla 5- ¿Qué valora más, la música, las matemáticas, 0 ambas por igual?

\begin{tabular}{c|cc} 
Música & $20 \%$ \\
\hline Matemáticas & $\vdots$ & $60 \%$ \\
Ambas & $\vdots$ & $124 \%$ \\
No contesta & $\vdots$ & $156 \%$ \\
\hline
\end{tabular}

Con respecto al ítem de si se siente satisfecho con su trabajo, se observa una gran diferencia en las respuestas obtenidas, ya que un 95\% de la muestra sí se siente satisfecha aunque dentro de estas personas también existe aproximadamente un 15\% que comenta, en el apartado de observaciones, que su trabajo mejoraría si disminuyera la ratio. En contraposición solamente un 5\% no se siente satisfecho con su trabajo.

En cuanto a la pregunta de si consideran que la enseñanza-aprendizaje mejora trabajando las dos áreas conjuntamente, sorprendentemente, el $88,8 \%$ de la muestra considera que ésta mejora trabajando las dos áreas conjuntamente.

\section{Discusión y conclusiones}

La mayor parte de la muestra (85\%) afirma que en sus centros es distinto profesor quien imparte ambas materias, siendo nimio el porcentaje que realiza las programaciones de forma conjunta $(12,5 \%)$ y nulo el que utiliza materiales y actividades conjuntas. Algo parecido sucede con el 15\% que declara ser el mismo profesor el que imparte ambas asignaturas: sólo el 15\% afirma realizar de forma conjunta las programaciones $y$ utilizar los mismos materiales y recursos para trabajar las dos disciplinas. Sin embargo llama poderosamente la atención el hecho de que el 90,9\% afirma emplear las mismas actividades para ambas materias, y el 81,8\% declara realizar la evaluación de forma interdisciplinar.

Curiosamente la gran mayoría de los entrevistados considera importante trabajar las matemáticas $\mathrm{y}$ la música de forma interdisciplinar, sin embargo, los resultados obtenidos en la investigación reflejan que la mayoría de los encuestados (88\%) no se sienten capacitados para trabajar ambas materias interdisciplinarmente, pues no se consideran suficientemente preparados. Aún así, siendo conscientes de que su trabajo podría ser más enriquecedor si tuviesen una formación más específica, casi la totalidad de la muestra (95\%) se siente satisfecha con su trabajo.

En cuanto a la metodología, aunque la totalidad de los sujetos afirman trabajar de forma interdisciplinar, apenas un pequeño porcentaje especifica los diferentes tipos de actividades que realizan en el día a día del aula. Además, apenas la mitad de la muestra destaca el trabajo basado en una metodología lúdica, y solo un 5\% de los docentes utiliza el trabajo por proyectos como pilar de su labor educativa. En cuanto a los recursos utilizados en el aula, destaca la diversidad de materiales (de creación propia, editoriales, audiovisuales, juegos) que se usan para llevar a cabo conjuntamente las áreas de matemáticas y música.

Con todo, debemos matizar ciertos datos que nos han llamado la atención. En primer lugar, es positivo el alto porcentaje que valora por igual la música y las matemáticas. Sin embargo, discrepamos del concepto que tiene el profesorado al sentirse preparados y satisfechos con la manera de abordar sus clases, teniendo en cuenta que los materiales y recursos que emplean son los típicos para las dos disciplinas: juegos de lógica e instrumentos musicales. Además, se aprecia poco interés en la elaboración propia de 
materiales y en la búsqueda de alternativas que promuevan el aprendizaje significativo, activo y participativo (palabras que utilizan la mayoría de los maestros y maestras a la hora de describir su metodología). Una manera de promover la motivación y el aprendizaje significativo del alumnado sería a través del trabajo por proyectos y de las salidas extraescolares.

Por último, los hallazgos extraídos a lo largo de este trabajo nos permiten realizar una serie de consideraciones finales o reflexiones para la mejora de las propuestas didácticas de los profesores interesados:

- El fin de esta investigación, no es solo mostrar la realidad escolar, sino también dar a conocer la necesidad de seguir trabajando y mejorando para llegar verdaderamente a una educación interdisciplinar.

- Los beneficios de la música y las matemáticas interdisciplinariamente son innumerables debido a que existe una conexión extraordinaria entre ambas. Una enseñanza adecuada puede ayudar a que los alumnos y alumnas consigan asociar conceptos. Como consecuencia, se obtendrá una educación integral y no fragmentada.

- Por último, para promover el aprendizaje significativo y la educación integral es muy importante la formación del profesorado y la renovación constante, tanto de las metodologías como de los materiales y recursos, adaptándose a las características y circunstancias de los pequeños y pequeñas.

\section{Referencias}

ALSINA, Ángel; ESCALADA, Cristina. Educación matemática en las primeras edades desde un enfoque sociocultural. Revista Aula de Infantil, Barcelona, n. 44, p. 26-30, 2008.

ÁLVAREZ, Diego; MOLLÁ, Cristina; ESTÉBANEZ, Noelia. LA SORDERA y la educación musical, 2009. Disponible en: <http://www. uam.es/personal_pdi/stmaria/resteban/LaSordera.pdf>. Acceso en: 24 jun. 2014.

ARBONÉS, Javier; MILRUD, Pablo. La armonía es numérica: música y matemáticas. Barcelona: RBA, 2011.

BONIL, Josep et al. El diálogo disciplinar, un camino necesario para avanzar hacia la complejidad. Investigación en la Escuela, Sevilla, n. 53, p. 83-97, 2004.

CANALS I TOLOSA, María Antonia. Vivir las matemáticas. Barcelona: Octaedro - Rosa Sensat, 2001.

CASTRO ROJAS, Adriana Lucía. Formación de docentes y educadores en educación infantil: una apuesta clave para el desarrollo integral de la primera infancia. Colombia: SM, 2008.

CHAO, Rocío. Fundamentos didácticos de los métodos pedagógicos del s. XX a la actualidad. Villalba: Conservatorio Profesional Municipal de Música, 2005.

CHA0, Rocío; MATO, María Dorinda; FERREIRO, Francisco Jesús. Music therapy in adolescent disruptive behavior. Procedia. Social and Behavioral Sciences, Netherlands, n.132, p. 608-614, 2014.

FAUVEL, John; FLOOD, Raymond. Music and mathematics: ¿from Pythagoras to Fractals? Oxford: Oxford University Press, 2003.

FERNÁNDEZ BRAVO, José Antonio. Didáctica de la matemática en educación infantil. Madrid: Mayéutica, 2006.

FERNÁNDEZ-CARRIÓN, Marta. Música y matemáticas: conexiones curriculares para un mayor éxito educativo, 2011. Disponible en: <http://recursostic.educacion.es/artes/>. Acceso en: 12 jul. 2014.

GÓMEZ MARTíN, Paco. Matemáticas y música en niños pequeños, 2012. Disponible en: <http://divulgamat2.ehu.es/ divulgamat15/index.php?option=com_alphacontent\&section=11\&category=67\&ltemid=67\&limitstart=20>. Acceso en: 04 jun. 2014 
GREGORIO GUIRLES, José Ramón. El constructivismo y las matemáticas. Sigma: Revista de Matemáticas, Vitoria, n. 2, p. 113-129, 2002.

IBARRIAGA, Íñigo. Música y matemáticas: de Schoenberg a Xenakis. Bilbao: Kuraia: Grupo de Música Contemporánea de Bilbao, 2010.

ILARI, Beatriz. Music and babies: a review of research with implications for music educators. Applications of Research in Music Education, Texas, n. 21, p. 17-26, 2002.

LEVITIN, Daniel. Tu cerebro y la música. Barcelona: RBA, 2011.

LEVITIN, Daniel; ÁLVAREZ FLÓREZ, José Manuel. Tu cerebro y la música: el estudio científico de una obsesión humana. Barcelona: RBA, 2008.

LIERN, Vicente; QUERALT, Tomás. Música y matemáticas: la armonía de los números. Badajoz: FESPM, 2008.

MATO, María Dorinda. Mejorar las actitudes hacia las matemáticas. Revista Galego-Portuguesa de Psicoloxía e Educación, A Coruña, v.18, n. 1, p. 1138-1663, 2010.

MATO, María Dorinda; MUÑOZ, Jesús Miguel. Evaluación de las actitudes hacia las matemáticas y el rendimiento académico. Revista de Ciencias Psicológicas, Montevideo, v. 4, n. 1, p. 27-40, 2010.

LAVALL, Esther. La música como herramienta interdisciplinar: un análisis cuantitativo en el aula de lengua extranjera de primaria. Revista de Investigación en Educación, Madrid, n. 10, p. 127-143, 2012.

THIESSEN, Diane. Exploring mathematics through literature: articles and lessons for Prekindergarten through grade 8. Reston: National Council of Teachers of Mathematics, Vigo, 2004.

WINKLER, István et al. Newborn infants detect the beat in music. Proceeding of the National Academy of Sciences of USA, Washington, v. 7, n. 106, p. 2468-2471, 2009.

Recebido en: 10.08.2014.

Aprobado en: 21.10.2014.

Rocío Chao Fernández es doctora en educación musical en el Departamento de Composición de la Universidad de Coruña, España. Sus líneas de investigación se centran en analizar los elementos que inciden en el aprendizaje musical y mejoran el rendimiento académico.

María Dorinda Mato Vázquez es doctora en educación matemática en el Departamento de Pedagogía y Didáctica de la Universidade da Coruña. Sus líneas de investigación se enmarcan en el estudio comparativo de las habilidades procedimentales que se enseñan en Ciencias de la Naturaleza y en Matemáticas.

Andrea $\mathbf{M}^{\mathrm{a}}$ López Chao es ingeniera superior de caminos, canales y puertos por la UDC y violonchelista. Sus líneas de investigación giran en torno a la interdisciplinariedad en Educación. 